\title{
Development of preference and spontaneous recovery in choice behavior with concurrent variable-interval schedules
}

\author{
JAMES E. MAZUR \\ Southern Connecticut State University, New Haven, Connecticut
}

\begin{abstract}
Pigeons pecked on two response keys that delivered reinforcers on a variable-interval schedule. The proportion of reinforcers delivered by one key was constant for a few sessions and then changed, and subjects' choice responses were recorded during these periods of transition. In Experiment 1, response proportions approached a new asymptote slightly more slowly when the switch in reinforcement proportions was more extreme. In Experiment 2, slightly faster transitions were found with higher overall rates of reinforcement. The results from the first session, after a switch in the reinforcement proportions, were generally consistent with a mathematical model that assumes that the strength of each response is increased by reinforcement and decreased by nonreinforcement. However, neither this model nor other similar models predicted the "spontaneous recovery" observed in later sessions: At the start of these sessions, response proportions reverted toward their preswitch levels. Computer simulations could mimic the spontaneous recovery by assuming that subjects store separate representations of response strength for each session, which are averaged at the start of each new session.
\end{abstract}

Compared with the numerous studies on "steadystate" choice behavior, there has been relatively little research on how preferences develop in the first place. In recent years, however, a number of experiments have examined choice behavior during periods of transitionperiods in which subjects' choice responses shift because of a recent change in the reinforcement contingencies for two different response alternatives (e.g., Couvillon \& Bitterman, 1985, 1988, 1991; Dreyfus, 1991; Kacelnik, Krebs, \& Ens, 1987). In a series of experiments with pigeons, my colleagues and I have used a procedure that allows us to obtain many "acquisition curves" from each subject (Bailey \& Mazur, 1990; Mazur, 1992; Mazur \& Ratti, 1991). In these experiments, each successive condition began with a period in which the reinforcement schedules associated with two response keys were identical, and then the development of preference was observed when one of the two keys suddenly began to deliver more reinforcers than the other key. In one experiment, the two reinforcement schedules were variable-interval (VI) schedules, and in other experiments, the alternatives were random-ratio (RR) schedules (in which each

This research was supported by Grant MH 38357 from the National Institute of Mental Health and by a Connecticut State University research grant. I thank Christopher D'Antonio, Jeff Colafrancesco, Margaret Nygren, and Peter Kelly for their help in different phases of the research. Correspondence should be addressed to J. E. Mazur, Psychology Department, Southern Connecticut State University, New Haven, CT 06515.

-Accepted by previous editor, Vincent M. LoLordo response on a given key had a fixed probability of being reinforced).

The results from these studies were used to test the different predictions made by some well-known models of transitional choice behavior, including Myerson's kinetic model (Myerson \& Hale, 1988; Myerson \& Miezin, 1980), Staddon's ratio-invariance model (Staddon, 1988; Staddon \& Horner, 1989), and the linear-operator model (Bush \& Mosteller, 1955). Mazur (1992) discussed the results of these experiments in detail, but to summarize them briefly, none of these models could predict the main trends in the data - that is, the models could not predict which conditions would have rapid transition rates and which would have slow ones. As one example, consider two conditions of an experiment with RR schedules (Mazur \& Ratti, 1991). In one condition, the reinforcement probabilities for the two response keys were .16 and .10 , and in another condition the reinforcement probabilities were .07 and .01 . How rapidly would subjects develop a preference for the higher probability of reinforcement in these two conditions? Myerson's kinetic model predicted equal rates of transition in the two conditions, because the reinforcement probabilities differ by the same amount (.06) in both. The ratioinvariance model and the simple linear-operator model predicted slightly faster acquisition of preference in the $.16 / .10$ condition. However, the actual results contradicted all of these predictions, with substantially faster acquisition in the $.07 / .01$ condition. In general, Mazur and Ratti (1991) found faster acquisition of preference when the ratio of the two alternative reinforcement probabilities was larger. 
Although these and other results from this series of experiments conflicted with the predictions of several different models of acquisition, Mazur (1992) showed that the results could be described quite well by a simple mathematical model that states that each response alternative, $i$, has a separate strength, $V_{i}$. Response strength increases each time the response is reinforced and decreases each time the response is not reinforced. For now, assume that $V_{i}$ can range from 0 to 1 , inclusive. The model states that each time a response is reinforced, $V_{i}$ increases as follows:

$$
\Delta V_{i}=r\left(1-V_{i}\right)
$$

where $\Delta V_{i}$ is the change in strength of $V_{i}$, and $r$ is a reinforcement parameter that can range from 0 to 1 . After each response that is not reinforced, the strength of the response decreases as follows:

$$
\Delta V_{i}=n\left(-V_{i}\right)
$$

where $n$ is a nonreinforcement parameter that can range from 0 to 1 .

These equations are very similar to those of the simple linear-operator model, except that in the linear-operator model the increments and decrements occur directly in $p_{i}$, the probability that response $i$ will occur. The equations of the ratio-invariance model also describe increments and decrements in $p_{i}$, not $V_{i}$. In the present model, however, a simple matching rule is used to translate from the independent strengths of two responses to the probability that one will occur:

$$
p_{1}=\frac{V_{1}}{V_{1}+V_{2}} \text {. }
$$

The model of Couvillon and Bitterman (1985) is similar to Equations 1-3, but it includes an additional equation, with two free parameters, to translate from values of $V$ to the response probability, $p_{1}$. This additional complexity may be necessary in some circumstances, but for the present purposes, the simpler form of Equations 1-3 will suffice. Computer simulations showed that the model's predictions were in agreement with all of the major results obtained by Mazur and colleagues (Bailey \& Mazur, 1990; Mazur, 1992; Mazur \& Ratti, 1991).

All of the models mentioned above use fairly simple mathematical equations to derive their predictions, and it would certainly be unrealistic to expect such simple models to predict all the subtle details of behavior in complex choice situations. However, Mazur and colleagues used fairly standard choice situations to ask some very basic questions: How is the rate of transition affected by different reinforcement probabilities, or by different reinforcement rates? If a mathematical model can predict anything about transitional choice behavior, it should be able to answer such basic questions correctly. The fact that not all models made the correct predictions suggests that it may be possible to distinguish between appropriate and inappropriate acquisition models of choice using very simple choice situations. For in- stance, the results discussed above showed that a model that assigned a separate strength $\left(V_{i}\right)$ to each alternative (Equations 1-3) made correct predictions, whereas two similar models that calculated only a single value of $p$ did not. Perhaps these results show that the calculation of separate strengths for every response alternative is an essential component of a successful model of choice acquisition.

One major purpose of the present research was to provide some additional data on transitional behavior in choice situations involving VI schedules. Numerous studies have examined choice behavior with such schedules, but very few have explored and reported the patterns of acquisition that precede steady-state performance (e.g., Catania, 1969; Dreyfus, 1991; Killeen, 1972). To the extent possible, the results will be used to compare and test the predictions of two different models of choice acquisition - the kinetic model (Myerson \& Miezin, 1980) and the model described by Equations 1-3.

A second purpose of this research was to demonstrate a limitation of both of these models and other comparable models, such as those mentioned above. Although these simple models may be able to predict, at least in some cases, the rates of acquisition within a single session, none predict the "spontaneous recovery" that occurred between sessions in the two experiments reported here. In this type of spontaneous recovery, response proportions at the beginning of a new session were not the same as those at the end of the previous session, but tended to revert toward their level of earlier sessions. This spontaneous recovery was large in size and followed an orderly pattern within and across conditions.

To try to account for this spontaneous recovery, I will start with a simple hypothesis-that responding at the start of each new session is a function of the events of the previous several sessions, not just of the immediately preceding session. To see how this hypothesis can be applied to cases of spontaneous recovery in choice behavior, consider a hypothetical example that is similar to some of the actual results obtained in the present experiments. Suppose the proportion of reinforcers delivered by the left response key has been .10 for three consecutive sessions, but in the fourth session it increases to .90 . The proportion of responses on the left key quickly increases, reaching .75 by the end of this session. However, at the beginning of the fifth session, the left-key response proportion exhibits spontaneous recovery, reverting to 45 .

I suggest that this decreased response proportion at the start of the new session reflects the mixture of reinforcement contingencies experienced in the previous few sessions. Now suppose that the left-key reinforcement proportion is again .90 in the fifth session. The proportion of left-key responses again increases, but drops to .60 at the start of the sixth session. I propose that this higher initial response proportion (.60 in the sixth session compared with .45 in the fifth) occurs because the reinforcement proportion of .90 has now been in effect in the two most recent sessions, not just one. In short, I hypothesize that this type of spontaneous recov- 
ery occurs because the subject's initial response proportion is based on some type of average of the consequences of the preceding several sessions.

Similar ideas about the effects of previous sessions have been proposed by Davison and Hunter (1979). Although this hypothesis is easy to describe, it remains to be seen whether it can account for the patterns of spontaneous recovery observed in an actual experiment. To make a concrete test of this hypothesis, the model described by Equations 1-3 will be modified so that the values of $V_{1}$ and $V_{2}$ at the start of each new session are not simply carried over from the end of the previous session, but depend on the experiences of the past several sessions. Computer simulations based on this model can then be compared with the experimental results.

\section{EXPERIMENT 1}

This experiment used a single VI 30 -sec schedule to program reinforcers on two response keys. Each time the VI schedule programmed a reinforcer, it was assigned to one of the two keys on a probabilistic basis. At the start of every condition, the proportion of reinforcers assigned to one key was $.10, .25$, or .40 , and the remaining reinforcers were assigned to the other key. After a few sessions, the reinforcement proportions were switched to $.90, .75$, or .60 (respectively), and they remained at these new values for the four subsequent sessions. Based on the abundant research related to Herrnstein's (1961, 1970) matching law, it is well known that once behavior stabilizes in this type of choice situation, subjects exhibit either matching or some degree of undermatching (Baum, 1974; Myers \& Myers, 1977). That is, response proportions may approximately equal the reinforcement proportions, or they may be slightly less extreme than the reinforcement proportions.

This experiment was conducted to determine how quickly response proportions approached their asymptotic levels with the three different reinforcement proportions, and to examine the patterns of spontaneous recovery in choice proportions across successive sessions. The kinetic model (Myerson \& Miezin, 1980) predicts identical rates of approach to asymptote in all three conditions because overall rates of reinforcement (summed across the two keys) were the same. That is, although a much greater shift in response proportions should occur in the .90 conditions than in the .60 conditions, the kinetic model predicted that subjects should approach this asymptote just as quickly. Computer simulations based on Equations 1-3 predicted a slightly slower approach to asymptote with the more extreme reinforcement proportions. As already explained, neither model (without additional assumptions) predicts spontaneous recovery of response proportions at the start of a new session.

\section{Method}

Subjects. The subjects were 6 White Carneaux pigeons that had previously served in Mazur's (1992) experiment and other exper- iments. They received most of their food in the experimental chambers, but were given additional food after each session, when necessary, to keep them at approximately $80 \%$ of their free-feeding weights.

Apparatus. The experimental chamber was $30 \mathrm{~cm}$ long, $30 \mathrm{~cm}$ wide, and $30.5 \mathrm{~cm}$ high. Recessed into the right side wall of the chamber, $22.8 \mathrm{~cm}$ above the floor, were three response keys, separated by $5.6 \mathrm{~cm}$; each measured $2.5 \mathrm{~cm}$ in diameter. The center key was not used in this experiment. Each of the two outside keys could be transilluminated by red or green light produced by two $2-W$ bulbs mounted behind the keys. A force of about $0.1 \mathrm{~N}$ was required to operate each key. A hopper that could present grain for a specified interval was situated $12 \mathrm{~cm}$ below the center key. When the grain was presented, the hopper was illuminated with white light from the two 2-W bulbs mounted above it. Two 2-W houselights were mounted above the Plexiglas ceiling of the chamber.

The chamber was enclosed in a sound-attenuating wooden box that had an exhaust fan for ventilation and for masking extraneous noises. An IBM-compatible personal computer was programmed in the Medstate language to control all stimuli and to record the responses.

Procedure. The pigeons all had had considerable experience with the procedure through their participation in a previous experiment, so no additional training was needed at the start of this experiment. The complete experiment included 24 conditions, each consisting of three or four pretransition sessions followed by one transition session and four posttransition sessions.

All sessions lasted for $30 \mathrm{~min}$. At the beginning of a session, the houselights came on, and the two response keys were illuminated-the left key red and the right key green. The houselights remained on for the entire session, except during reinforcement periods. Every effective peck on either key produced a 0.1 -sec darkening of both keys as feedback. If a response resulted in reinforcement, the houselights and keylights were turned off, and the hopper was illuminated and raised to present grain for $2.5 \mathrm{sec}$. After reinforcement, the houselights and keylights were again illuminated. At the end of a session, all chamber lights were turned off. This procedure was used throughout the experiment, and the only differences among conditions were the reinforcement proportions for the two keys, as described below.

In all sessions, reinforcers were assigned by a single VI $30-\mathrm{sec}$ schedule: Once per second, if no reinforcer was currently assigned to one of the two keys, there was a .0333 probability that a reinforcer would be assigned to one of the two keys. Assignment to the left or right key was determined by a pseudorandom sequence that assured that the actual proportion of reinforcers delivered by each key was close to the scheduled proportion. Once a reinforcer was assigned to a key, no further reinforcers were assigned to either key until that reinforcer was delivered. The procedure also included a 2-sec changeover delay (COD); that is, 2 sec had to elapse after each changeover response before a response could be reinforced. If a reinforcer was assigned to a key, it was delivered following the first post-COD response on that key.

In the pretransition sessions that began each condition, the probability of assigning a reinforcer to the left key was (in different conditions) $.10, .25, .40, .60, .75$, or .90 . The pretransition sessions were followed by one transition session in which the same reinforcement probability was in effect for the first $6 \mathrm{~min}$, after which the probabilities for the two keys switched. The switched probabilities remained in effect for the rest of the transition session and for the four posttransition sessions. For example, if the left-key reinforcement probability was .10 for the pretransition sessions and for the first $6 \mathrm{~min}$ of the transition session, the leftkey probability then switched to .90 for the rest of the condition.

For each subject, each of the six probabilities was repeated in four different conditions, for a total of 24 conditions. The order of reinforcement probabilities was counterbalanced across subjects. 


\section{Results}

Performance in the transition sessions. For simplicity, the key with the higher reinforcement proportion after the transition will be called the "rich key" for that condition (even though this key had a lower reinforcement proportion before the transition). This experiment therefore included eight conditions each with rich-key reinforcement proportions of $.60, .75$, and .90 . Figure 1 shows the patterns of transition for these three different reinforcement proportions, averaged across subjects, replications, and key locations. The $y$-axis plots $p_{1}$, the proportion of responses on the rich key. The center panel of this figure shows the results from the transition sessions, divided into 103-min blocks. Recall that the switch in reinforcement proportions occurred after the first 6 min of a transition session (i.e., at the end of the second block, as indicated by the dashed line). This figure shows that response proportions changed rapidly after the switch in reinforcement proportions, and by the end of the transition session the subjects showed a clear preference for the rich key.

For comparison, the left panel of Figure 1 shows the results from the last three blocks of the final pretransition session, and the right panel of the figure shows the results from the last three blocks of the final posttransition session. These two panels show that after several sessions with the same reinforcement proportions, there was still considerable undermatching in all conditions. For example, when the reinforcement proportion was $.90, p_{1}$ averaged about .77 at the end of the final posttransition session.

Computer simulations were used to compare these results with the predictions of Equations 1-3. Because they focused on the results of the single transition ses-

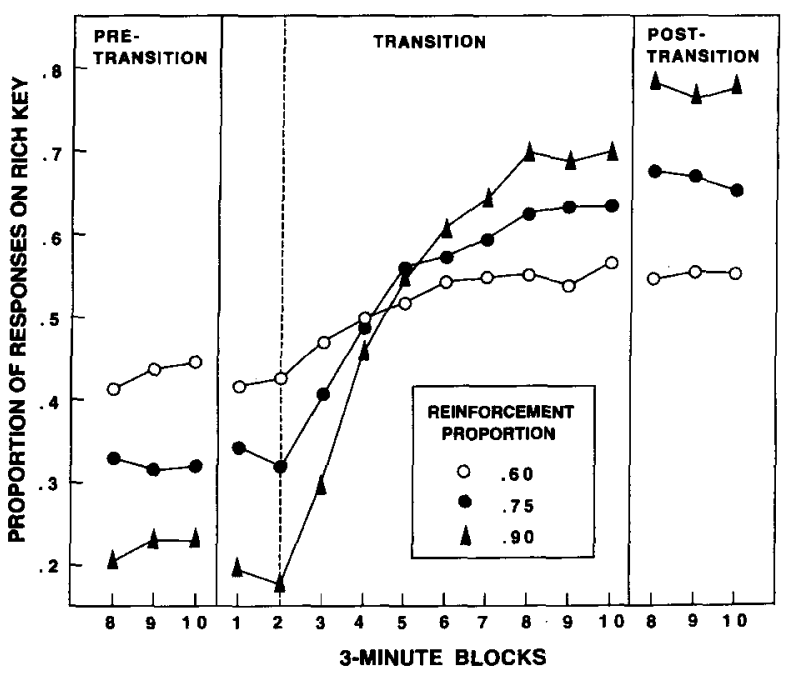

Figure 1. Mean response proportions with the three different reinforcement proportions in Experiment 1 are shown for part of the last pretransition sessions, the transition sessions, and part of the last posttransition sessions. The dashed line marks the time the reinforcement proportions for the two keys were switched.

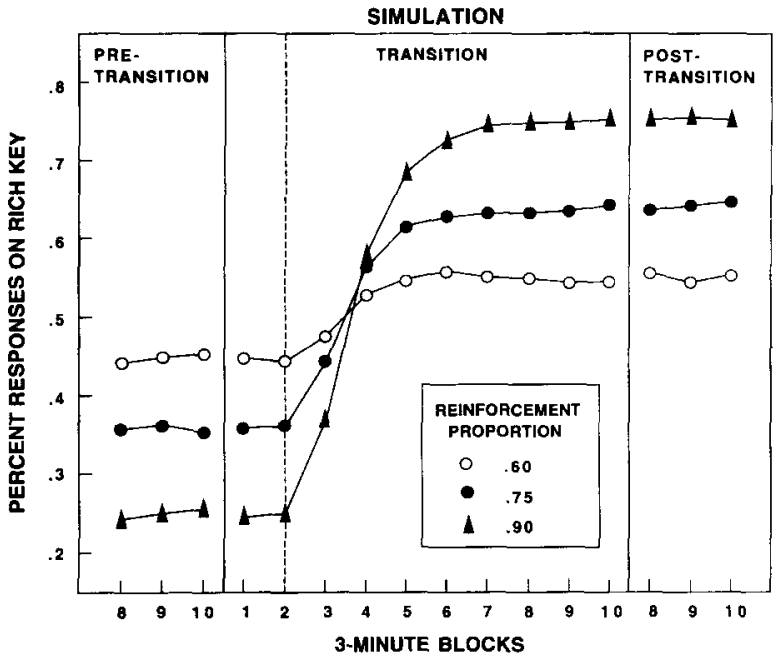

Figure 2. Results of computer simulations for Experiment 1 are plotted in the same form as that for the actual data in Figure 1. Each curve is the mean of 300 simulations based on Equations 1-3, with $r=$ .0005 and $n=.005$. See text for details.

sion, these initial simulations were not modified to account for spontaneous recovery, but employed the same procedures and parameter values used by Mazur (1992) to generate successful predictions for previous experiments of this type. The reinforcement parameter, $r$, was set at .0005 , and the nonreinforcement parameter, $n$, was set at .005 . A constant response rate of one response per second was used, which roughly approximated the average response rate in the experiment. The COD was simulated by not reinforcing either of the first two responses on one key after a changeover from the other key. The computer program conducted a series of simulations, each consisting of four pretransition sessions and one transition session. Simulated responses were chosen on a probabilistic basis according to Equation 3, using the current values of $V_{1}$ and $V_{2}$. A VI 30 -sec schedule assigned reinforcers to the two response alternatives on a probabilistic basis. Because responses and reinforcers were both chosen probabilistically, repeated simulations with the same reinforcement proportions often produced fairly different acquisition curves. To reduce the variability and determine the average results predicted by the model, 300 simulations were conducted for each of the three reinforcement probabilities used in Experiment 1.

Figure 2 shows the means of these 300 simulations for each reinforcement probability, presented in the same form as that for the actual data in Figure 1. The simulated response proportions changed quickly after the switch in reinforcement proportions, and with the parameter values used, they approached asymptote somewhat more quickly than the subjects' actual response proportions. Nevertheless, the similarity of the actual and simulated results can be seen by comparing Figures 1 and 2.

To assess the reliability of the group means, standard errors of the mean were calculated for every data point 


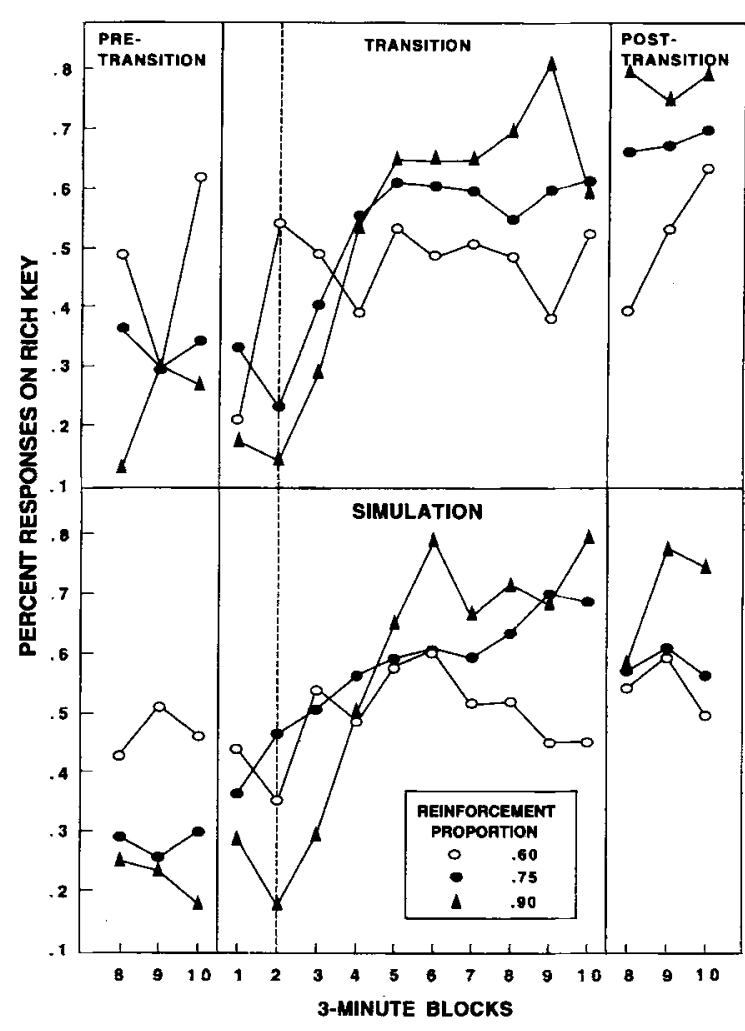

Figure 3. The top panels show typical results from 3 different subjects, with one example for each of the three reinforcement proportions used in Experiment 1 . In each case, the results are from a single condition, not averaged over replications. The bottom panels show three typical examples of individual computer simulations, one for each of the three reinforcement proportions. Parameters for the simulations were the same as those for Figure 2.

in Figure 1; these standard errors were consistently small, ranging from .014 to .027 . These small standard errors were a consequence of the many replications conducted for each reinforcement proportion, but results from individual subjects in individual sessions were much more variable. To illustrate this point, the top half of Figure 3 shows three typical examples of choice proportions from individual subjects in individual 3-min blocks. (Data from 3 different subjects are shown.) For comparison, the bottom half of Figure 3 shows the results of three individual computer simulations based on Equations 1-3. It should be clear that for the actual results (Figure 1) as well as the computer simulations (Figure 2), the relatively smooth acquisition functions emerged only through the averaging process, and individual acquisition functions were quite variable.

As already explained, the kinetic model predicts equal rates of approach to asymptote in all three conditions. In contrast, the computer simulations based on Equations 1-3 predicted a small difference among the conditions (a difference not obvious from visual inspection of Figure 2): They predicted roughly a $10 \%$ faster rate of approach toward asymptote in the .60 conditions than in the .90 conditions, with the .75 conditions falling in between. The predictions of these two models are therefore not very different, and it may be difficult to distinguish between them. However, notice that at the end of the transition session, $p_{1}$ was approximately .70 in the .90 conditions, which is substantially below the proportion of .77 reached four sessions later. On the other hand, in the .60 conditions, $p_{1}$ reached its final level of about .55 by Block 7 of the transition session. This comparison suggests a faster rate of approach to asymptote in the .60 conditions.

To facilitate a comparison of the rates of approach to asymptote in the three different conditions, the results from the transition session were normalized by plotting them as a percentage of the total distance between the final pretransition and posttransition response proportions. Each subject's response proportion during Block 2 of the transition session (the last $3 \mathrm{~min}$ before the transition) was used as a starting point. Response proportions during the last three blocks of the final posttransition session (as is shown in the right panel of Figure 1) were used as an estimate of asymptotic performance. For example, suppose a subject's response proportion was .20 before the transition and .80 at the end of the condition. This subject's response proportion would be $33 \%$ of the way to asymptote when it reached .40 , and $67 \%$ of the way to asymptote when it reached .60 . Such percentages were calculated for each subject, and the group results are shown in Figure 4 for the three different reinforcement proportions. This figure shows that, on a percentage basis, the rate of transition was fastest in the .60 conditions. A repeated measures analysis of variance (ANOVA) was conducted with the normalized data shown in Figure 4. There was a significant effect of 3 -min blocks $[F(7,35)=37.47, p<.01]$. The effect of reinforcement proportion was not significant $[F(2,10)=$ $2.44]$, nor was the block $\times$ reinforcement proportion interaction $[F(14,70)=0.44]$. However, to conduct a more sensitive test of the specific prediction of Equa-

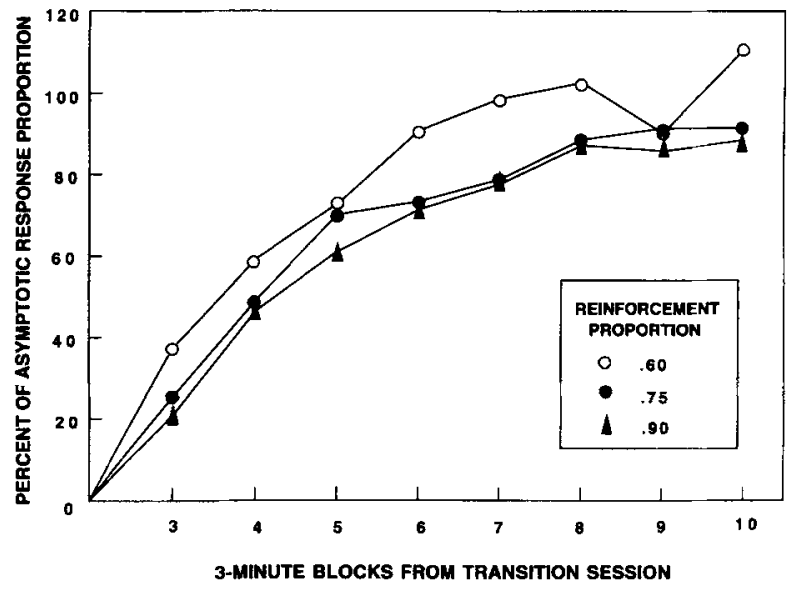

Figure 4. Mean response proportions from the transition sessions of Experiment 1 are plotted as a percentage of the distance between the final pretransition response proportions and the final posttransition response proportions. 


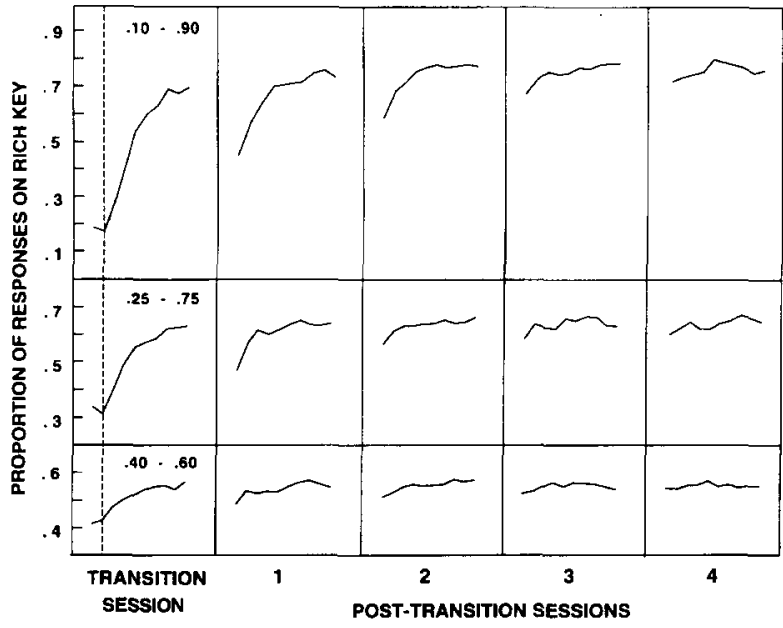

Figure 5. Mean response proportions with the three different reinforcement proportions in Experiment 1 are shown for the transition sessions and the four posttransition sessions. The dashed line marks the time the reinforcement proportions for the two keys were switched.

tions 1-3 (that the rate of transition should be fastest in the .60 conditions and slowest in the .90 conditions), a planned comparison was used. This planned comparison used the data from the first four 3-min blocks after the switch in reinforcement proportions (Blocks 3-6), during which $p_{1}$ was changing most rapidly and differences among conditions should be largest. The planned comparison showed a significant trend in the predicted direction $[F(1,10)=5.33, p<.05]$.

Spontaneous recovery across sessions. Figure 5 shows the group results with the three different reinforcement proportions for the transition session and the four posttransition sessions. As in the previous figures, $p_{1}$ is plotted in 3-min blocks. This figure shows that at the start of each posttransition session, $p_{1}$ was lower than that at the end of the preceding session. This spontaneous recovery was evident with all three reinforcement proportions. It was largest in the first posttransition session and became progressively smaller in subsequent sessions.

A new series of computer simulations was conducted using Equations 1-3, with one change. Instead of simply carrying over the values of $V_{i}$ from one session to the next, at the start of each session, $V_{i}$ (for each response, 1 and 2) was set to a weighted mean of its value at the end of the five previous sessions. In other words, the events of the preceding five sessions each contributed to the beginning values of $V_{i}$ for each new session. A weighted mean was used because it was assumed that the events of the most recent sessions would have more influence on choice than would the events of earlier sessions. More specifically, $V_{i}$ for the preceding session had a weight of $0.3, V_{i}$ for the fifth most recent session had a weight of 0.1 , and the sessions in between had intermediate weights (summing to 1.0 ). In all other respects, the computer simulations were the same as those described previously, and 100 simulations were conducted for each reinforce- ment proportion. The results of these simulations are shown in Figure 6, and the similarity between the simulations and the actual results can be seen by comparing Figures 5 and 6 .

Notice that in these simulations, the same weights were assigned to previous sessions regardless of which reinforcement proportions were in effect in those sessions. If this approach is correct, it follows that the relative amounts of spontaneous recovery should be the same for all three reinforcement proportions. To test this assumption, Figure 7 compares the relative amounts of spontaneous recovery with the three different reinforcement proportions. The data in this figure were normalized by using the same measure as that in Figure 4, to show the percent change from the pretransition values of $p_{1}$ to the final posttransition values. In Figure 7, these percentages are plotted for the first 3-min blocks of the four successive posttransition sessions. This figure shows that when the differences between the pretransition and posttransition response proportions are taken into account, the relative magnitudes of the spontaneous recovery were quite similar to the three different reinforcement proportions.

\section{Discussion}

This experiment was similar to a previous study (Mazur, 1992, Experiment 2), but in the earlier study the reinforcement proportion was always .50 for each key before the switch. After the switch, the reinforcement proportion for one key was $.60, .75$, or .90 , and the rates of approach toward asymptote were not significantly different among these three conditions. In the present experiment, more extreme shifts in the reinforcement proportions and more replications were used, and results showed slightly but significantly faster rates of approach

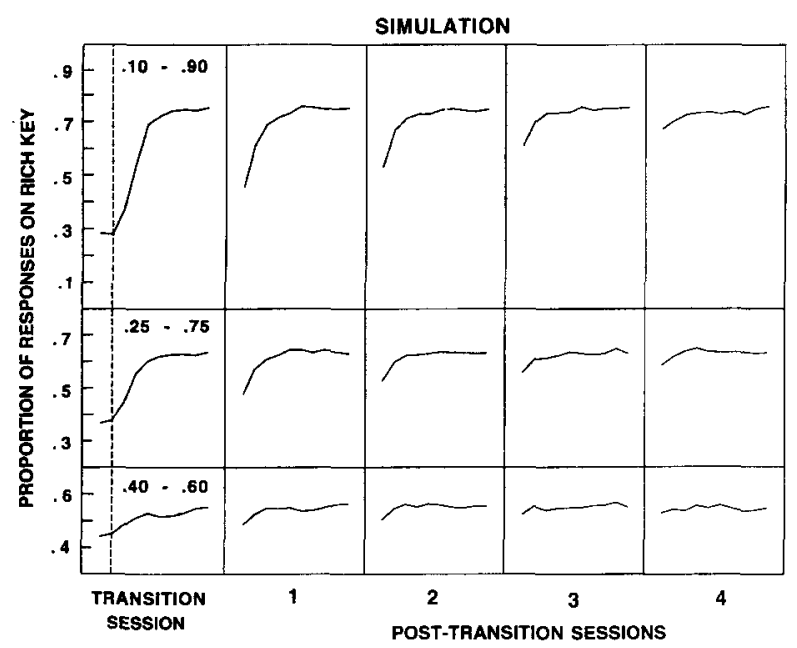

Figure 6. Results of computer simulations for Experiment 1 are plotted in the same form as that for the actual data in Figure 5. Each curve is the mean of 100 simulations based on Equations 1-3, plus the additional rule that, at the start of each session, $V_{1}$ and $V_{2}$ were based on weighted means of their final values from the five previous sessions. See text for details. 


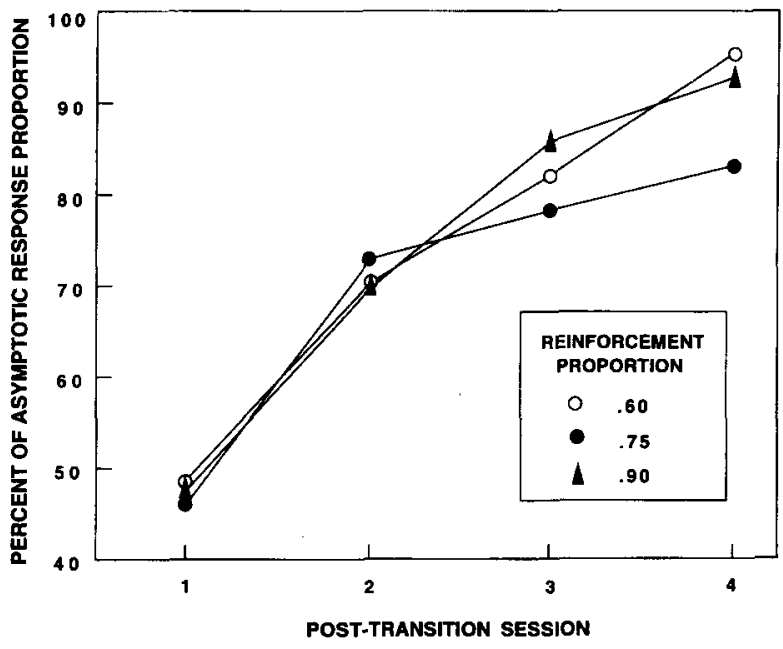

Figure 7. The mean response proportions from the first 3-min blocks of the posttransition sessions are plotted as a percentage of the distance between the final pretransition response proportions and the final posttransition response proportions.

to asymptote when the shift in reinforcement proportions was less extreme (shifting from .40 to .60 rather than from .10 to .90 ). This effect, though small, was not consistent with the kinetic model, which predicts an equally rapid approach toward asymptote in all three conditions. This small effect was predicted, however, by computer simulations based on Equations 1-3.

The spontaneous recovery obtained in the posttransition sessions was not predicted by either model in its simplest form, nor by any of the other acquisition models mentioned in the introduction. In addition, this type of spontaneous recovery is not predicted by the "cumulativeeffects model" recently developed by Davis, Staddon, Machado, and Palmer (1993). All of these models assume that after a change in the reinforcement proportions, response proportions will move fairly steadily toward their new levels, with no major discontinuities from the end of one session to the beginning of the next. The results shown in Figure 5 make it clear, however, that this assumption is not correct. In one way or another, some additional features must be added to these models if they are to predict the spontaneous recovery of previous response tendencies at the start of a new session.

Over the years, a number of different theories of spontaneous recovery have been proposed (e.g., Estes, 1955; Pavlov, 1927; Robbins, 1990; Skinner, 1950). Of course, spontaneous recovery has usually been defined as a reappearance of a previously extinguished response, not a return to a previously preferred response, and the term has seldom been applied to behavior in choice situations (but see Beale, 1970). Some of these theories are not easily applied to choice behavior, and, in any case, the relative merits of the different theories will not be evaluated here. However, the simulations presented in Figure 6 show that the patterns of spontaneous recovery found in this experiment are not difficult to predict if it is assumed that they are the result of a simple averaging process.

Additional simulations based on Equations 1-3 were conducted, and they showed that the patterns of spontaneous recovery observed in the experiment can be mimicked by using a variety of different averaging rules, including some that are almost surely too simple to be realistic. For example, one set of simulations gave equal weights (.2 each) to the values of $V_{i}$ from the last five sessions. The results of these simulations were very similar to those in Figure 6, except that the magnitude of the spontaneous recovery was larger in all cases. Thus, the patterns of spontaneous recovery observed in this experiment could be simulated reasonably well by using Equations 1-3 and several different averaging rules. If this spontaneous recovery was indeed the result of some sort of averaging of the events of previous sessions, the exact nature of the averaging process cannot be specified very precisely with this limited set of data.

\section{EXPERIMENT 2}

Another variable that has been extensively studied in steady-state choice behavior, but not during periods of transition, is the overall rate of reinforcement. Experiment 2 compared the rates of acquisition when the schedule assigning reinforcers to the two keys was VI $30 \mathrm{sec}$, VI $60 \mathrm{sec}$, or VI $90 \mathrm{sec}$. Each condition began with a reinforcement proportion of .5 for both keys, and then it switched to .9 for one of the two keys.

The kinetic model makes a clear prediction for this experiment: The rate of transition should be directly proportional to the overall rate of reinforcement (Myerson \& Miezin, 1980). Therefore, compared with the VI 30 -sec conditions, the rate of transition should be twice as slow in the VI 60-sec conditions and three times as slow in the VI 90 -sec conditions.

The predictions of Equations 1-3 are less clear. A series of computer simulations found that, depending on the parameter values used for $r$ and $n$, this model predicts no major differences in transition rates among the conditions, a slight advantage for longer VI schedules, or a slight advantage for shorter VI schedules. Shorter VI schedules were more likely to have faster transition rates if the simulations assumed that response rates were faster with shorter VI schedules (which is often the case; see Catania \& Reynolds, 1968; de Villiers \& Herrnstein, 1976). Although these predictions are not very specific, they are clearly different from those of the kinetic model. They suggest that differences in the overall reinforcement rate have at most a small effect on the rate of transition - the differences in transition rates should not be as large as the differences in reinforcement rates.

\section{Method}

Subjects and Apparatus. The 6 subjects from Experiment 1 served in this experiment, and the same apparatus was used. 


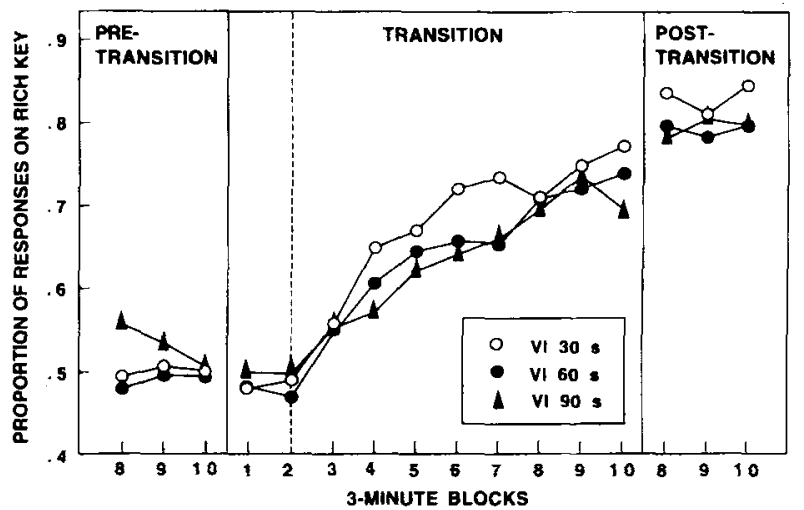

Figure 8. Mean response proportions with the three different variableinterval (VI) schedules in Experiment 2 are shown for part of the last pretransition sessions, the transition sessions, and part of the last posttransition sessions. The dashed line marks the time the reinforcement proportion for the rich key switched from .50 to .90 .

Procedure. Except as explained below, the procedure was the same as that in Experiment 1: Each condition included three or four pretransition sessions, one transition session, and four posttransition sessions. Individual sessions were also similar to those in Experiment 1: All sessions lasted for $30 \mathrm{~min}$, a single VI schedule programmed the reinforcers for the two response keys, and a 2 -sec COD was in effect.

The experiment consisted of 12 conditions. In all conditions, the reinforcement probability was .5 for both keys in the pretransition sessions and in the first $6 \mathrm{~min}$ of the transition session. For the remainder of the transition session and for the posttransition sessions, the reinforcement probability was .9 for one key and .1 for the other key. Four conditions used a VI $30-\mathrm{sec}$ schedule, four conditions used a VI 60 -sec schedule, and four used a VI 90 -sec schedule. Of the four conditions with each VI schedule, the left key had the higher reinforcement proportion in two conditions, and it had the lower reinforcement proportion in the other two conditions. The order of key assignments and VI schedules was counterbalanced across subjects.

\section{Results}

Figure 8 shows the patterns of transition for these three different reinforcement rates, averaged across subjects, replications, and key locations. The $y$-axis plots $p_{1}$, the proportion of responses on the rich key. As in Figure 1 , the three panels show the results from the end of the last pretransition session, from the entire transition session, and from the end of the last posttransition session. Similar to Experiment 1, individual transition functions from individual subjects were quite variable, but because of the large number of replications, standard errors of the mean were small, ranging from .016 to .045 for the data points in Figure 8. Similar standard errors were obtained for the data from subsequent sessions shown in Figure 9.

With all three reinforcement rates, $p_{1}$ increased rapidly after the switch in reinforcement proportions. The rate of transition was fastest with VI $30 \mathrm{sec}$ and slowest with VI $90 \mathrm{sec}$, but the differences among the three conditions were not large. A two-way repeated measures ANOVA showed a significant effect of 3 -min blocks $[F(8,40)=$
$41.70, p<.01]$ and a significant effect of VI schedule $[F(2,10)=4.64, p<.05]$. The block $\times$ schedule interaction was not significant $[F(16,80)=1.65]$. To make a more specific test of the prediction that $p_{1}$ would be highest in the VI 30-sec condition and lowest in the VI 90 -sec condition, a planned comparison was used. This planned comparison showed a significant effect of schedule size $[F(1,10)=7.33, p<.05]$.

These results show that the rates of transition were a function of the rates of reinforcement, but not to the degree predicted by the kinetic model. The kinetic model predicts that the rate of transition to asymptote should proceed according to the following exponential equation:

$$
p_{1}(t)=(0.5-K) \cdot e^{(-t m)}+K,
$$

where $p_{1}(t)$ is the proportion of responses on the rich key at time $t$, the number of minutes since the switch in reinforcement proportions. $K$ is the asymptotic response proportion, $e$ is the base of the natural logarithm, and $m$ is a learning rate parameter that should be proportional to the overall rate of reinforcement. A least-squares curve-fitting procedure was applied to the group averages from the transition sessions to obtain estimates of $K$ and $m$ for each of the three reinforcement rates. For the VI 30 -sec, VI 60-sec, and VI 90 -sec conditions, respectively, the best-fitting estimates of $K$ were $.76, .75$, and .75 , and the best-fitting estimates of $m$ were $.17, .11$, and .09. These estimates of $m$ confirm the visual impression that the transition rates did not differ as much as predicted by the kinetic model. For instance, the model predicts that $m$ should be three times as large with VI $30 \mathrm{sec}$ as with VI $90 \mathrm{sec}$, whereas it was actually only about twice as large.

Figure 9 shows the group means for the transition sessions and the four posttransition sessions, again plotted in 3-min blocks. As in Experiment 1, decreases in $p_{1}$ were

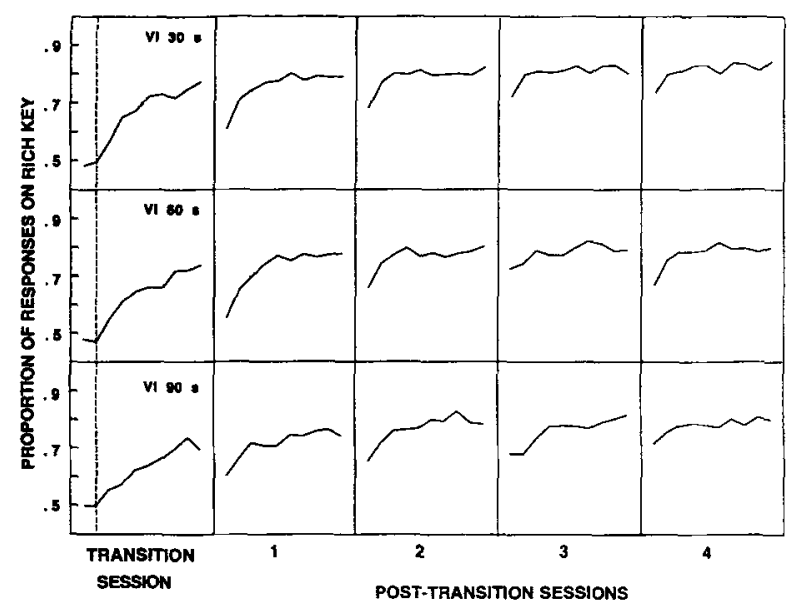

Figure 9. Mean response proportions with the three different variableinterval (VI) schedules in Experiment 2 are shown for the transition sessions and the four posttransition sessions. The dashed line marks the time the reinforcement proportion for the rich key switched from .50 to .90 . 
seen at the start of the posttransition sessions. The amounts of spontaneous recovery were similar with the three different reinforcement rates. They were largest in the first posttransition session and generally became progressively smaller in subsequent sessions.

Although the experiment was not designed for this purpose, it should be possible to observe spontaneous recovery in the first three (or four) sessions of each condition, during which the reinforcement proportion was .50 for each key. These sessions were immediately preceded by four posttransition sessions from the previous condition in which the reinforcement proportion for one key was .90 . The amounts of spontaneous recovery in these sessions cannot be directly compared with those seen in Figure 9, because besides the switch in reinforcement proportions, there was also a change in reinforcement rates from one condition to the next. Nevertheless, the data from these sessions provide an opportunity to observe a case in which the spontaneous recovery should consist of a return to a more extreme, rather than less extreme, preference for one key. This experiment included 12 cases per subject in which there was such a switch from a reinforcement proportion of .90 to .50 , and Figure 10 presents the mean results from all of these cases. Response proportions are shown for the final posttransition session of one condition and the four pretransition sessions of the next condition. There was substantial spontaneous recovery in the second pretransition session, but much less in the third and fourth sessions.

\section{Discussion}

The kinetic model predicts that after a switch in reinforcement proportions with VI schedules, the rate of transition should be proportional to the overall rate of reinforcement. This prediction also follows from the general idea that each reinforcer provides information about the current reinforcement contingencies, and the faster this information is delivered, the faster the subject's behav-

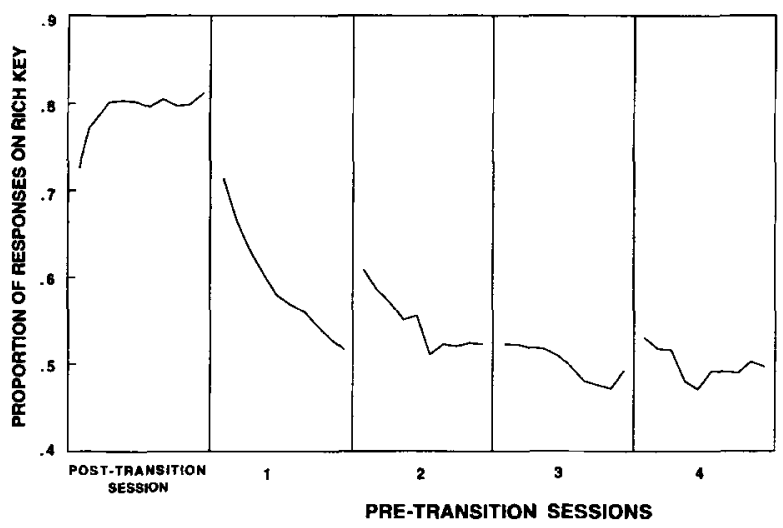

Figure 10. Mean response proportions, averaged across all conditions, are shown for the final postransition session of one condition and the pretransition sessions of the next condition. The reinforcement proportion for the rich key was .90 in the posttransition sessions and .50 in the pretransition sessions. ior should change. However, the results of this experiment did not support these predictions. As the overall rate of reinforcement increased by a factor of three, the rate of transition did increase, but not by a factor of three. The rate of transition was only about twice as fast with VI $30 \mathrm{sec}$ as with VI $90 \mathrm{sec}$.

As discussed above, Equations 1-3 predicted (depending on the parameter values) few or no differences in transition rates among the three conditions. Although there was a statistically significant effect of VI size, the differences shown in Figure 8 are small, so at least in this limited sense, the results were consistent with Equations 1-3. However, because the predictions are not specific, the results cannot be viewed as strong evidence either for or against this model.

As in Experiment 1, substantial spontaneous recovery in response proportions was observed in the posttransition sessions. Computer simulations similar to those used for Experiment 1 were run, which began each new session with values of $V_{1}$ and $V_{2}$ that were averages of the values from the five previous sessions. One set of simulations assigned greater weights to the values of $V_{i}$ from more recent sessions (the same weights used for Figure 6), and another set gave equal weights to the last five sessions. The simulations are not shown here, but they were similar to those displayed in Figure 6. As before, both sets of simulations captured the main trends in the results - decreased spontaneous recovery across successive sessions and about equal levels of spontaneous recovery with all three reinforcement rates. Once again, the simulations showed that the observed patterns of spontaneous recovery can be readily modeled by using some fairly simple assumptions about the influence of previous sessions on current choice behavior.

The results in Figure 10, which showed the changes in response proportions between the end of one condition and the start of the next, demonstrated that spontaneous recovery in choice behavior need not always appear as a shift toward indifference, but can consist of a reversion to a more extreme response proportion. However, the spontaneous recovery in Figure 10 disappeared more quickly over successive sessions than that shown in Figure 9. There are at least two possible reasons for this faster disappearance. First, besides the tendency to revert to the response proportions of previous sessions, there may be some tendency to begin a session closer to indifference (i.e., with $p_{1}$ closer to .5). These two tendencies would sum when there was a switch to a more extreme reinforcement proportion (as in Figure 9), but would counteract each other when there was a switch to a less extreme reinforcement proportion (as in Figure 10). Second, the transitions shown in Figure 10, which occurred at the end of one condition and the start of the next, did not provide an ideal test of spontaneous recovery, because there was always a change in the overall rate of reinforcement at this point. The subjects therefore had two cues to signal that the reinforcement contingencies had changed-the change in reinforcement proportions and 
the change in reinforcement rates. The combination of these two cues may have produced a faster disappearance of the spontaneous recovery.

\section{GENERAL DISCUSSION}

These results, particularly those from Experiment 1, join others in showing that a simple mathematical model (Equations 1-3) can predict the gross features of transitional choice behavior, at least within a single session (Bailey \& Mazur, 1990; Mazur, 1992; Mazur \& Ratti, 1991). The model has been applied with reasonable success to choices involving both ratio and interval schedules. The model is based on some very basic assumptions-that the strength of each response alternative is independently increased by reinforcement and decreased by nonreinforcement, and that choice behavior depends on the current relative strengths of the two alternatives.

Whereas this model has been shown to predict transition rates in a variety of situations, it is not true that virtually any set of assumptions about choice behavior can predict these results. Several models of comparable or greater complexity, including the kinetic model (Myerson \& Miezin, 1980), the ratio-invariance model (Staddon, 1988), and the linear-operator model (Bush \& Mosteller, 1955), could not account for the results of previous experiments. The two experiments reported here made this point again, by showing that the kinetic model did not accurately predict the rates of transition in the different conditions.

In summary, some starting assumptions about transitional choice behavior yield predictions that are consistent with the data, and others do not. Yet, even if Equations 1-3 offer a useful set of initial assumptions, it should be clear that these equations, by themselves, are too simple to account for many of the details of choice behavior. For example, Mazur (1992) noted that these equations treat successive responses as separate and independent choices, so the equations cannot account for the temporal or sequential patterns of responses that have often been observed in choice situations (e.g., Hinson \& Staddon, 1983; Silberberg, Hamilton, Ziriax, \& Casey, 1978).

The present experiments illustrated another limitation of Equations 1-3 (and of the other models as well): The models include no provision for the spontaneous recovery of previous response proportions at the start of a new session. I suggest that in this case, the oversimplification is the assumption that each response, $i$, has only a single strength, $V_{i}$, which is carried over from one session to the next. The results of these two studies indicate that response proportions at the start of a session reflect the independent influences of the past several sessions. One way to model these separate influences, as already described, is to assume that the subject stores a separate value of $V_{i}$ for each successive session, and its response proportion at the start of a new session is some sort of average of the values from the past few sessions. The computer simulations described above showed that it is easy to model the main features of this spontaneous recovery, even with unrealistically simple assumptions about the averaging process (such as giving equal weight to each of the previous five sessions, as was done in some of the simulations). It is almost certainly more realistic to give greater weight to more recent sessions, as was done for the simulations shown in Figure 6.

Superficially, this averaging process may appear similar to the exponentially weighted moving average (EWMA) used by Killeen (1982) in his incentive theory. However, the resemblance is only superficial, because the type of moving average Killeen employed predicts only a steady increase (or decrease) in response proportions over time for the conditions used in the present experiments. Without modification, an EWMA cannot predict the discontinuities in response proportions observed from the end of one session to the beginning of the next. To predict such discontinuities, a model must give more weight to the events of previous sessions at the start of each new session than at the end of the session. The model described here accomplished this by making the starting values of $V_{1}$ and $V_{2}$ dependent on their values in the past several sessions, and by then letting these values change within a session according to Equations 1 and 2 . There are certainly other ways to achieve such discontinuities in response proportions, but a simple, smoothly changing EWMA is not one of them.

The "averaging model" of spontaneous recovery described here does have some similarity to Estes's (1955) model of stimulus fluctuations. Estes assumed that the environment contains numerous stimulus elements, each of which may or may not be associated with a given response. The associations between stimulus elements and responses are created through reinforcement and broken through nonreinforcement. Estes also assumed that not all stimulus elements are present every session, so that after one session of conditioning and one session of extinction, some stimulus elements will be associated with the response and others will not. Because of random stimulus fluctuations, the next session is likely to include a mixture of associated and nonassociated stimulus elements, so spontaneous recovery (a partial recovery of responding) will be observed. Estes did not extend his analysis to choice behavior, but if we assume that each stimulus element can be associated with either of two response alternatives, the model can readily account for the patterns of spontaneous recovery observed in the present experiments. For example, if one key delivers a high proportion of the reinforcers for several consecutive sessions, each session more and more of the stimulus elements will become conditioned to this response, so less and less spontaneous recovery of the other response will be observed.

Although the assumptions of Estes's model and the averaging model are somewhat different, for now their similarities will be emphasized. Both models assume that behavior at the start of a session reflects the combined influence of several different measures of response strength (separate stimulus elements in Estes's 
model; separate estimates of $V$ in the averaging model). Perhaps this is the most basic conclusion that can be drawn from the evidence for spontaneous recovery in choice situations: Postulating a single representation for the strength of each response alternative (whether it is $V, p$, or some other measure) is not sufficient. Such an approach cannot explain why response proportions at the start of a new session move toward indifference in some situations and away from indifference in other situations, depending on the reinforcement contingencies that prevailed in previous sessions. As we have seen, however, it is easy to model this type of spontaneous recovery by assuming that there are several independent representations for each response alternative. Yet although the present results suggest that a model involving multiple representations of response strength is needed, they do not specify the exact nature of this model (because the general patterns of spontaneous recovery observed here can be approximated by using a variety of different assumptions). Additional work on spontaneous recovery in choice behavior will be necessary before we can state more precisely how the events of the past several sessions combine to affect responding at the start of a new session.

\section{REFERENCES}

BAILEY, J. T., \& MazUr, J. E. (1990). Choice behavior in transition: Development of preference for the higher probability of reinforcement. Journal of the Experimental Analysis of Behavior, 53, 409422.

BAUM, W. M. (1974). On two types of deviation from the matching law: Bias and undermatching. Journal of the Experimental Analysis of Behavior, 22, 231-242.

BEALE, I. L. (1970). The effects of amount of training per reversal on successive reversals of a color discrimination. Journal of the Experimental Analysis of Behavior, 14, 345-352.

BUSH, R. R., \& MOSTELLER, F. (1955). Stochastic models for learning. New York: Wiley.

CATANIA, A. C. (1969). Concurrent performances: Inhibition of one response by reinforcement of another. Journal of the Experimental Analysis of Behavior, 12, 731-744.

Catania, A. C., \& ReYnolds, G. S. (1968). A quantitative analysis of the responding maintained by interval schedules of reinforcement. Journal of the Experimental Analysis of Behavior, 11, 327383.

Couvillon, P. A., \& Bitterman, M. E. (1985). Analysis of choice in honeybees. Animal Learning \& Behavior, 13, 246-252.

Couvillon, P. A., \& BitTerman, M. E. (1988). Compound-component and conditional discrimination of colors and odors by honeybees: Further tests of a continuity model. Animal Learning \& Behavior, 16, 67-74.

Couvillon, P. A., \& Bitterman, M. E. (1991). How honeybees make choices. In L. J. Goodman \& R. C. Fisher (Eds.), The behaviour and physiology of bees (pp. 116-130). Wallingford: CAB International.

Davis, D. G. S., Staddon, J. E. R., Machado, A., \& Palmer, R. G. (1993). The process of recurrent choice. Psychological Review, 100, 320-341.
Davison, M. C., \& Hunter, I. W. (1979). Concurrent schedules: Undermatching and control by previous experimental conditions. Journal of the Experimental Analysis of Behavior, 32, 233-244.

DE Villiers, P. A., \& Herrnstein, R. J. (1976). Toward a law of response strength. Psychological Bulletin, 83, 1131-1153.

DREYFUS, L. R. (1991). Local shifts in relative reinforcement rate and time allocation on concurrent schedules. Journal of Experimental Psychology: Animal Behavior Processes, 17, 486-502.

EsTeS, W. K. (1955). Statistical theory of spontaneous recovery and regression. Psychological Review, 62, 145-154.

HERRNSTEIN, R. J. (1961). Relative and absolute strength of response as a function of the frequency of reinforcement. Journal of the Experimental Analysis of Behavior, 4, 267-272.

HerRnstein, R. J. (1970). On the law of effect. Journal of the Experimental Analysis of Behavior, 13, 243-266.

Hinson, J. M., \& Staddon, J. E. R. (1983). Hill-climbing by pigeons. Journal of the Experimental Analysis of Behavior, 39, 25-47.

KACELNIK, A., KREBS, J. R., \& ENS, B. (1987), Foraging in a changing environment: An experiment with Starlings (Sturnus vulgaris). In M. L. Commons, A. Kacelnik, \& S. J. Shettleworth (Eds.), Quantitative analyses of behavior: Vol. 6. Foraging (pp. 63-87). Hillsdale, $\mathrm{NJ}$ : Erlbaum.

KILLEEN, P. (1972). A yoked-chamber comparison of concurrent and multiple schedules. Journal of the Experimental Analysis of Behavior, 18, 13-22.

KIlleen, P. (1982). Incentive theory. In D. J. Bernstein (Ed.), Nebraska Symposium on Motivation, 1981: Vol. 29. Response structure and organization (pp. 169-216). Lincoln: University of Nebraska Press.

MAZUR, J. E. (1992). Choice behavior in transition: Development of preference with ratio and interval schedules. Journal of Experimental Psychology: Animal Behavior Processes, 18, 364-378.

MazUR, J. E., \& RaTTI, T. A. (1991). Choice behavior in transition: Development of preference in a free-operant procedure. Animal Learning \& Behavior, 19, 241-248.

MYERS, D. L., \& MYERS, L. E. (1977). Undermatching: A reappraisal of performance on concurrent variable-interval schedules of reinforcement. Journal of the Experimental Analysis of Behavior, 27, 203-214.

Myerson, J., \& Hale, S. (1988). Choice in transition: A comparison of melioration and the kinetic model. Journal of the Experimental Analysis of Behavior, 49, 291-302.

Myerson, J., \& Miezin, F. M. (1980). The kinetics of choice: An operant systems analysis. Psychological Review, 87, 160-174.

Pavlov, I. P. (1927). Conditioned reflexes (G. V. Anrep, Trans.). London: Oxford University Press.

RoBbins, S. J. (1990). Mechanisms underlying spontaneous recovery in autoshaping. Journal of Experimental Psychology: Animal Behavior Processes, 16, 235-249.

Silberberg, A., Hamilton, B., Ziriax, J. M., \& Casey, J. (1978). The structure of choice. Journal of Experimental Psychology: Animal Behavior Processes, 4, 368-398.

SkINNER, B. F. (1950). Are theories of learning necessary? Psychological Review, 57, 193-216.

STADDON, J. E. R. (1988). Quasi-dynamic choice models: Melioration and ratio invariance. Journal of the Experimental Analysis of Behavior, 49, 303-320.

StADDON, J. E. R., \& HoRner, J. M. (1989). Stochastic choice models: A comparison between Bush-Mosteller and a source-independent reward-following model. Journal of the Experimental Analysis of Behavior, 52, 57-64.

(Manuscript received September 8, 1993; revision accepted for publication April 28, 1994.) 